\title{
Unsteady aerodynamics modeling using Volterra series expansed by basis function
}

\author{
Senlin Chen $^{1}$, Zhenghong Gao ${ }^{1}$ \\ ${ }^{1}$ School of Aeronautics, Northwestern Polytechnical University, Xi'an 710072, China
}

\begin{abstract}
Unsteady aerodynamics modeling must accurately describe nonlinear aerodynamic characteristics in addition to unsteady aerodynamic characteristics. The Volterra series has attracted increasing attention as a powerful tool for nonlinear system modeling. It is essential to incorporate the influence of the second-order Volterra kernel or higher-order kernels to build a nonlinear unsteady aerodynamics model. The main difficulty in the identification of higher-order kernels is that the number of parameters to be identified increases exponentially with the order of a kernel. This paper expands the Volterra kernels with the four-order B-spline wavelet on the interval as the basis function, converts the problem into the solution of low-dimensional equations, and obtains a stable solution. A nonlinear unsteady aerodynamics model is built by identifying the second-order and third-order kernels of the lift, drag, and pitching moment coefficients of the NACA0012 airfoil. Then the model is verified at different reduced frequencies using CFD.
\end{abstract}

\section{Introduction}

Aerodynamics exhibit evident nonlinear unsteady hysteresis characteristics when a complex flow of shock waves, flow separation, vortices, etc. occurs in a flow field [1]. At this point, aerodynamics depend not only on the current exercise state but also on their time history [2], and the conventional aerodynamic derivative modeling method becomes difficult to apply [3]. Thus, the study of unsteady aerodynamic modeling methods is required.

Several methods have been proposed for unsteady aerodynamic modeling [4-6]. The Volterra series [7] method is a mathematical model that describes the nonlinear time-invariant system proposed by Italian mathematician Volterra. There have been a few studies on aerodynamic modeling in recent years [8-10]. The Volterra series method is a series model whose ability to describe the nonlinear characteristics of a system increases with the number of kernels involved. Most existing studies only use the first-order Volterra kernel or the truncated second-order Volterra kernel [10]. Hence, such methods are only suitable for aerodynamic modeling with linear or weak nonlinearities and are difficult to apply for strongly nonlinear aerodynamic modeling. Second-order or even higher-order kernels are rarely used because the identification of higher-order kernels is difficult. For example, second-order kernel identification based on an impulse response is extremely complicated and time consuming [9]. As the number of parameters to be identified increases exponentially with kernel order, the introduction of higher-order kernels leads to the so-called dimensionality disaster, which results from solving high-dimensional ill-posed equations.

Regarding the difficulties in Volterra kernel identification, Silva [11] and Balajewicz [12] proposed a truncation method that preserves the main diagonal of a high-order kernel or its nearby elements. As this approach neglects a large number of coupling factors in the Volterra kernel, it reduces the ability of the Volterra series to express nonlinearities. The other method is the basis function method [13, 14], which uses a set of basis functions to expand the Volterra kernel, thereby transforming the problem into the estimation of limited expansion coefficients.

In this paper, the Volterra kernel is expanded using the B-spline wavelet on the interval (BSWI) as a basis function. The identification of Volterra kernels is converted into a small number of wavelet coefficients by utilizing its good approximation characteristics. This considerably reduces the number of parameters to be identified. Finally, the ability of the Volterra series to describe nonlinear unsteady aerodynamics is verified by predicting the lift coefficient, drag coefficient, and pitching moment coefficient of the NACA0012 airfoil in plunging motion at transonic speed.

\section{Volterra series}

The Volterra series theory [15] shows that the input output relation of any continuous-time, causal, time-invariant, fading memory, and nonlinear system can be formulated as an infinite sum of multidimensional convolution integrals. 


$$
\begin{gathered}
y(t)=y_{0}+\sum_{n=1}^{\infty} \int_{0}^{t} \int_{0}^{t} L \int_{0}^{t} h_{n}\left(\tau_{1}, L, \tau_{n}\right) \bullet \\
\prod_{k=1}^{n} u\left(t-\tau_{k}\right) \mathrm{d} \tau_{1} L \mathrm{~d} \tau_{n}
\end{gathered}
$$

where $y(t)$ is the response of a nonlinear system to input $u(t), y_{0}$ is the steady-state response, $h_{1}(\tau)$ is the first-order Volterra kernel and the linear unit impulse response, and $h_{n}\left(\tau_{1}, \tau_{2}, L, \tau_{n}\right)$ is the $n$ th-order Volterra kernel. Without loss of generality, higher-order kernels $h_{n}(n \geq 2)$ are supposed to be symmetric, namely, $h_{n}\left(\tau_{1}, \tau_{2}, L, \tau_{n}\right)=h_{n}\left(\tau_{\pi(1)}, \tau_{\pi(2)}, L, \tau_{\pi(n)}\right)$, where $\pi(1), \pi(2), L, \pi(n)$ are all the permutations of integers 1 through $n$ [15].

Silva [11] showed that for a linear system, higher-order kernels are zero and the system is completely characterized by the first-order kernel. For a nonlinear system, the first-order kernel reflects the linear part of the system and higher-order kernels represent the nonlinear part of the system. Even though the Volterra series is the sum of infinite terms, the nonlinear influence for an actual physical system decays rapidly with the increase in kernel order. Thus, the first few terms can typically represent the system quite accurately [16]. This study is limited to the first three order kernels; hence, Eq. (1) reduces to

$$
y(t)=y_{0}+y_{1}(t)+y_{2}(t)+y_{3}(t)
$$

where

$$
\begin{aligned}
y_{1}(t)= & \int_{0}^{t} h_{1}(\tau) u(t-\tau) \mathrm{d} \tau \\
y_{2}(t)= & \int_{0}^{t} \int_{0}^{t} h_{2}\left(\tau_{1}, \tau_{2}\right) u\left(t-\tau_{1}\right) u\left(t-\tau_{2}\right) \mathrm{d} \tau_{1} \mathrm{~d} \tau_{2} \\
y_{3}(t)= & \int_{0}^{t} \int_{0}^{t} \int_{0}^{t} h_{3}\left(\tau_{1}, \tau_{2}, \tau_{3}\right) u\left(t-\tau_{1}\right) u\left(t-\tau_{2}\right) \times \\
& u\left(t-\tau_{3}\right) \mathrm{d} \tau_{1} \mathrm{~d} \tau_{2} \mathrm{~d} \tau_{3}
\end{aligned}
$$

where $y_{1}(t), y_{2}(t), y_{3}(t)$ are the responses of the first-order kernel, second-order kernel, and third-order kernel, respectively.

It should be noted that this identification problem is ill-posed in that the objective is to determine the structure of the system from input and output measurements [17]. Therefore, to obtain stable kernel estimation, a regularization method must be used to solve the least square problem. In this study, we use truncated singular value decomposition.

\section{Volterra kernel identification based on BSWI}

\subsection{B-spline wavelet on the interval (BSWI)}

On a bounded interval, the BSWI basis function has the good characteristics of semi-orthogonality, symmetry, and smoothness [18]. The BSWI function is $\phi_{m, k}^{j}(t) m, k, j \in \phi$, where $\phi$ is the set of all integers, $m$ is the order of the B-spline, and $j$ is the dilation index that determines the scale or resolution of the function. $k \in\left[-m+1,2^{j}-1\right]$ is the translation index that provides the position of the function. The four-order BSWI $\phi_{4, k}^{3}(t)$, where $j=3$, is shown in Figure 1 . Concrete expressions are provided in [18].

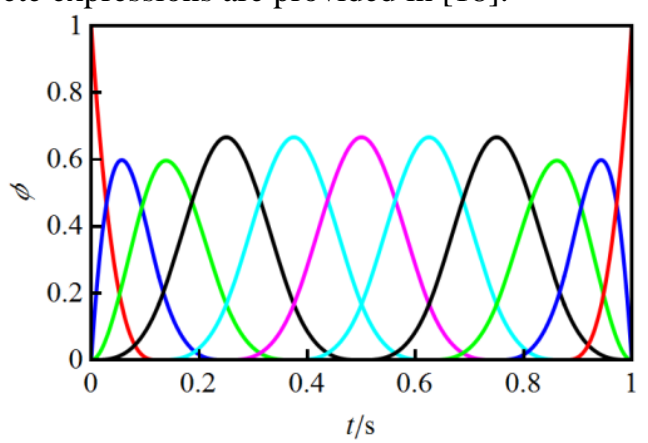

Figure 1. Four-order B-spline wavelet on the interval $(j=3$ )

The basis function in the two-dimensional case is the tensor product of two one-dimensional basis functions

$$
\Phi_{m,\left(k_{1}, k_{2}\right)}^{j}(x, y)=\phi_{m, k_{1}}^{j}(x) \phi_{m, k_{2}}^{j}(y)
$$

where $\Phi_{j,(k, m)}^{(s, v)}(x, y)$ is the two-dimensional basis function. Similarly, the basis function in the three-dimensional case is the tensor product of three one-dimensional basis functions, the complete expression for which is provided in [13].

\subsection{Volterra kernel identification}

The first-order kernel part of Eq. (3) is discretized for the identification of the first-order Volterra kernel. The response at $t_{n}=2^{-j} n(n=1,2, L, N)$ is

$$
y_{1, j}\left(t_{n}\right)=\int_{0}^{t_{n}} h_{1}(\tau) u\left(t_{n}-\tau\right) \mathrm{d} \tau
$$

where $N$ is the number of sample data.

The first-order kernel can be represented as a linear combination of basis functions

$$
h_{1, j_{1}}(\tau)=\sum_{k=1-m}^{2^{j_{1}}-1} \alpha_{m, k}^{j_{1}} \phi_{m, k}^{j_{1}}(\tau)
$$

Substituting Eq. (6) into Eq. (5), the following simplified matrix form can be obtained:

$$
\boldsymbol{y}_{1, j}=\boldsymbol{M}_{\boldsymbol{\alpha}_{j_{1}}} \boldsymbol{\alpha}_{j_{1}}
$$

where $\boldsymbol{\alpha}_{j_{1}}$ is a vector of $\alpha_{m, k}^{j_{1}}$ in Eq. (6), namely, the coefficient of the basis function that represents the first-order kernel. $\boldsymbol{M}_{\boldsymbol{\alpha}_{j_{1}}}$ is a coefficient matrix of $\boldsymbol{\alpha}_{j_{1}}$; it is composed of $\phi_{m, k}^{j_{1}}(\tau)$ and $u\left(t_{n}-\tau\right)$ in Eq. (5). Eq. (7) is solved for $\boldsymbol{\alpha}_{j_{1}}$ to obtain $h_{1}$ from Eq. (6).

The second-order kernel part of Eq. (3) is discretized for the identification of the second-order Volterra kernel. The response at $t_{n}$ is

$$
\begin{gathered}
y_{2, j}\left(t_{n}\right)=\int_{0}^{t_{n}} \int_{0}^{t_{n}} h_{2}\left(\tau_{1}, \tau_{2}\right) u\left(t_{n}-\tau_{1}\right) \bullet \\
u\left(t_{n}-\tau_{2}\right) \mathrm{d} \tau_{1} \mathrm{~d} \tau_{2}
\end{gathered}
$$


The second-order kernel can be represented as a linear combination of two-dimensional basis functions.

$$
h_{2, j_{2}}\left(\tau_{1}, \tau_{2}\right)=\sum_{k_{1}, k_{2}=1-m}^{2^{j_{2}}-1} \alpha_{m,\left(k_{1}, k_{2}\right)}^{j_{2}} \phi_{m, k_{1}}^{j_{2}}\left(\tau_{1}\right) \phi_{m, k_{2}}^{j_{2}}\left(\tau_{2}\right)
$$

Substituting Eq. (9) into Eq. (8), the following simplified matrix form can be obtained:

$$
\boldsymbol{y}_{2, j}=\boldsymbol{M}_{\boldsymbol{\alpha}_{j_{2}}} \boldsymbol{\alpha}_{j_{2}}
$$

where $\boldsymbol{\alpha}_{j_{2}}$ is a vector of $\alpha_{m,\left(k_{1}, k_{2}\right)}^{j_{2}}$ in Eq. (9), namely, the coefficient of the basis function that represents the second-order kernel. $\boldsymbol{M}_{\boldsymbol{\alpha}_{j_{2}}}$ is a coefficient matrix of $\boldsymbol{\alpha}_{j_{2}}$; it is composed of $\phi_{m, k_{1}}^{j_{2}}\left(\tau_{1}\right) \phi_{m, k_{2}}^{j_{2}}\left(\tau_{2}\right)$ and $u\left(t_{n}-\tau_{1}\right)$ $u\left(t_{n}-\tau_{2}\right)$ in Eq. (8). Eq. (10) is solved for $\boldsymbol{\alpha}_{j_{2}}$ to obtain $h_{2}$ from Eq. (9).

The identification of the third-order kernel is similar to that of the second-order kernel. Hence, it is not repeated here.

\section{Aerodynamics modeling with Volterra series}

Considering the plunge motion of the NACA0012 airfoil at transonic speed as an example, this study verified the ability of the Volterra series to predict nonlinear unsteady aerodynamics and the effectiveness of BSWI basis functions for Volterra kernel identification.

\subsection{Input}

Input selection and design is extremely important in system identification, and it directly determines the accuracy of parameter identification. In Volterra kernel identification, first-order kernel identification is a linear system identification problem and there are several input signals such as impulse [11] and sweep frequency [19]. Higher-order kernel identification is a nonlinear system identification problem, and currently, there is no universal input signal. This study used an orthogonal phase-optimized multisine input designed by Morelli[20].

$$
u=\sum_{n=1}^{M} A_{n} \sin \left(\frac{2 \pi n t}{T}+\phi_{n}\right)
$$

where $u$ is the input, $A_{n}$ is the amplitude, $T$ is the time length of input excitation, $\phi_{n}$ is the phase shift, and $M$ is the total number of available harmonic frequencies. The first step is to select the time period, $T$, for the input, which determines the smallest harmonic frequency resolution, $\Delta f=1 / T \mathrm{~Hz}$, and the limit on the minimum frequency, $f_{\min } \geq 2 / T$. The second step is select the frequency band, $\left[f_{\min }, f_{\max }\right] \mathrm{Hz}$, for the input. The total number of frequencies is $M=\operatorname{fix}\left[\left(f_{\max }-f_{\min }\right) / \Delta f\right]+1$, where "fix" indicates rounding to the nearest integer toward zero. The third step is select the input power spectrum by specifying and the amplitude of the input to determine the amplitude, $A_{n}$, of each component. The last step is to assign the starting values for phase angles, $\phi_{n} \in[-\pi, \pi]$, and then apply an optimization algorithm to adjust $\phi_{n}$ to achieve the minimum relative peak factor (RPF) for the input.

$$
\operatorname{RPF}(u)=\frac{[\max (u)-\min (u)] / 2}{\sqrt{2\left(u^{T} u\right) / N}}
$$

where $N$ is the number of sample data. The orthogonal phase-optimized multisine input is a sum of selected harmonic sinusoids, and it is orthogonal. This input can reflect the coupling of different frequencies, and it is suitable for the identification of nonlinear systems [14].

When the airfoil is in plunge motion, only the angle of attack changes. Considering the angle of attack as an independent variable, the relationship between the angle of attack and displacement is

$$
s(t)=-V \int_{0}^{t} \tan [u(\tau)] \mathrm{d} \tau
$$

where $V$ is the freestream velocity, $u$ is the angle of attack, and $s$ is the displacement of the airfoil. The output used for identification is calculated through CFD. The conditions are, $M_{\infty}=0.8$ and $R e=7.2 \times 10^{6}$, and a rigid dynamic grid is used.

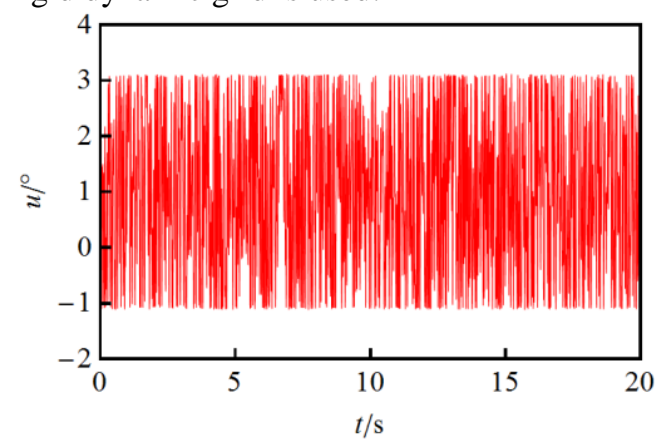

Figure 2. Orthogonal phase-optimized multisine input

\subsection{Volterra kernel identification of aerodynamics}

The orthogonal phase-optimized multisine input is considered, and then the outputs with different amplitude inputs are used to separate the responses generated by various order kernels to identify them separately. The initial angle of attack, $\alpha_{0}=1^{\circ}$, is selected as the reference state, the input duration is $T=20 \mathrm{~s}$, the minimum and maximum frequencies are $f_{\min }=3 / 20 \mathrm{~Hz}$ and $f_{\max }=30 \mathrm{~Hz}$ respectively, and the amplitude of each component of the input is $A_{n}=2 / \sqrt{M}$. The input can be seen in Figure 2. The first three order kernels identification results of the lift coefficient, drag coefficient, and pitching moment coefficient are shown in Figure 3-Figure 5, where the third-order kernels, $h_{3}\left(\tau_{1}, \tau_{2}, \tau_{3}\right)$, are plotted at $\tau_{3}=0.01 \mathrm{~s}$. 


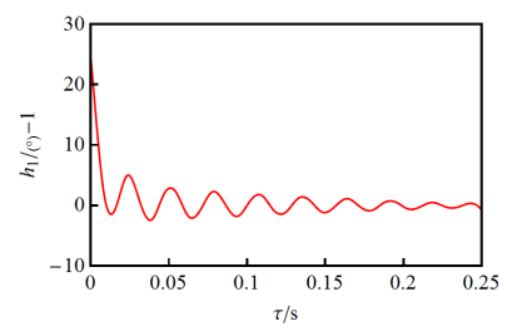

(a) $h_{1}(\tau)$

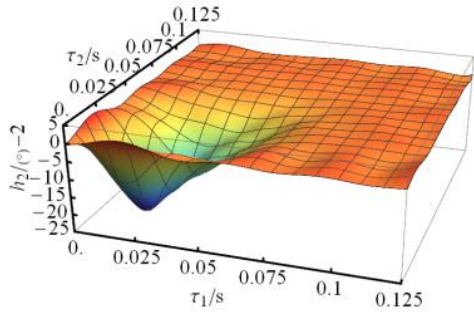

(b) $h_{2}\left(\tau_{1}, \tau_{2}\right)$

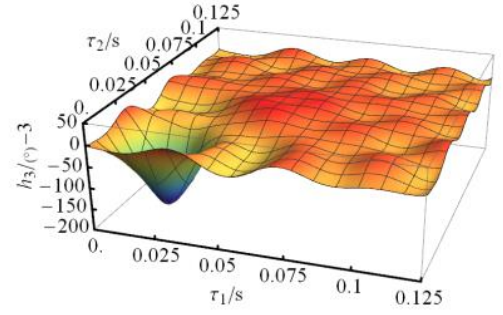

(c) $h_{3}\left(\tau_{1}, \tau_{2}, 0.01\right)$

Figure 3. Volterra kernel of lift coefficient when angle of attack $\alpha_{0}=1^{\circ}$

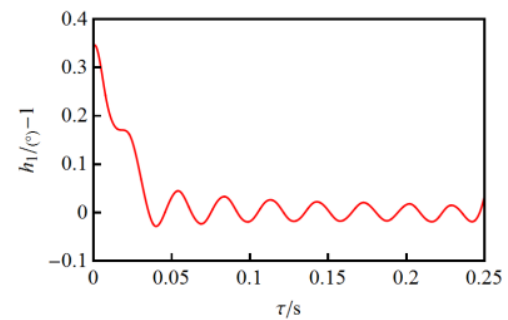

(a) $h_{1}(\tau)$

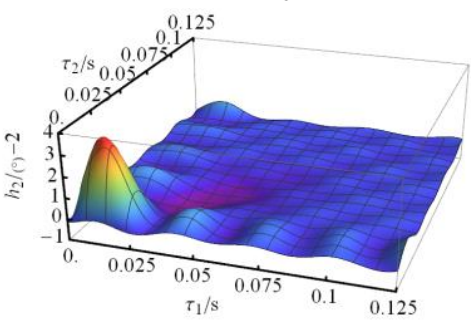

(b) $h_{2}\left(\tau_{1}, \tau_{2}\right)$

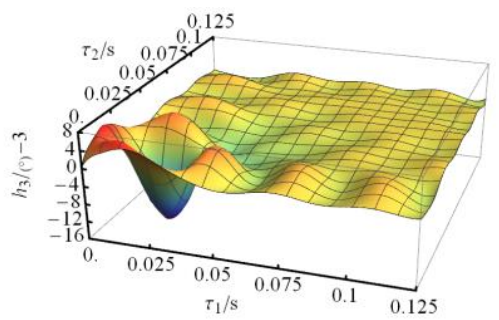

(c) $h_{3}\left(\tau_{1}, \tau_{2}, 0.01\right)$

Figure 4. Volterra kernel of drag coefficient when angle of attack $\alpha_{0}=1^{\circ}$

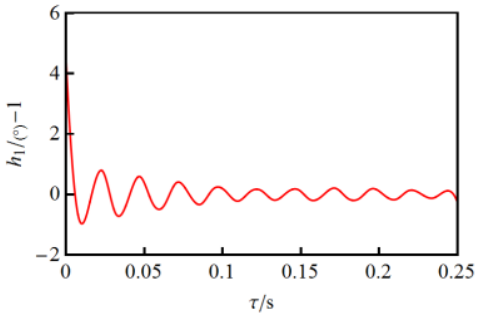

(a) $h_{1}(\tau)$

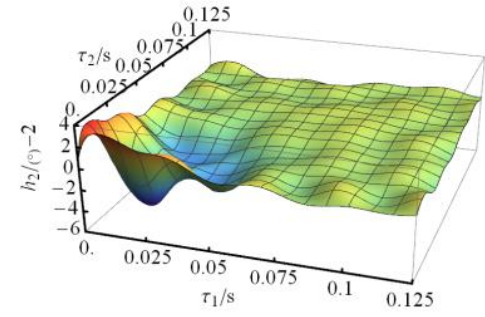

(b) $h_{2}\left(\tau_{1}, \tau_{2}\right)$

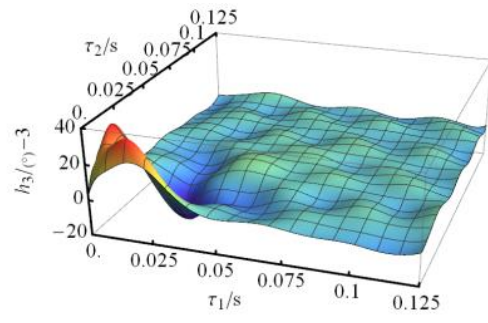

(c) $h_{3}\left(\tau_{1}, \tau_{2}, 0.01\right)$

Figure 5. Volterra kernel of pitching moment coefficient when angle of attack $\alpha_{0}=1^{\circ}$

\subsection{Transonic aerodynamics validation}

The aerodynamics model has been established as we have obtained the Volterra kernels. The next step is to validate the ability of the Volterra series to predict nonlinear unsteady aerodynamics. The displacement of the airfoil in plunge motion at the initial angle of attack, $\alpha_{0}=1^{\circ}$, is

$$
\begin{aligned}
& s(t)=0.5236(\cos (23.612 t)-1) \\
& s(t)=0.1309(\cos (94.448 t)-1)
\end{aligned}
$$

The corresponding reduced frequencies are 0.05 and 0.1 .

The lift coefficient, drag coefficient, and pitching moment coefficient calculated by the Volterra series and CFD are shown in Figure 6 and Figure 7. When the

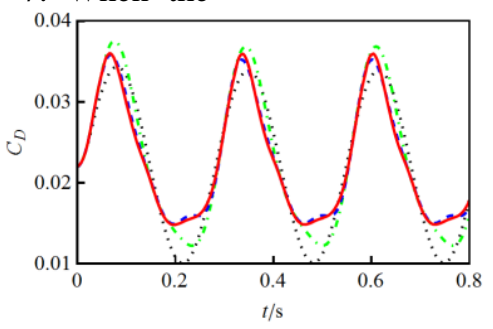

(b) $C_{D}$ reduced frequency is 0.05 , the aerodynamics calculated by the first-order kernel reflect the basic features. However, there are clear errors. The system is nonlinear at this time. Prediction accuracy improves considerably with the introduction of the second-order kernel. Prediction results become extremely close to CFD results after the third-order kernel is introduced. When the reduced frequency is 0.1 , the errors in aerodynamics calculated by the first-order kernel are reduced, and prediction accuracy is gradually improved after the introduction of higher-order kernels.

In summary, higher-order kernels characterize the nonlinearity of aerodynamics. To accurately predict aerodynamics, it is necessary to incorporate the influence of higher-order kernels based on the first-order kernel. (a) $C_{L}$

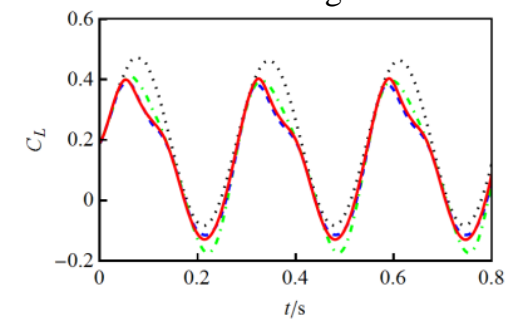

$\cdots \cdots \cdots h_{1}=--=-h_{1}+h_{2}$

$\cdot-\cdot \cdot-h_{1}+h_{2}+h_{3}$

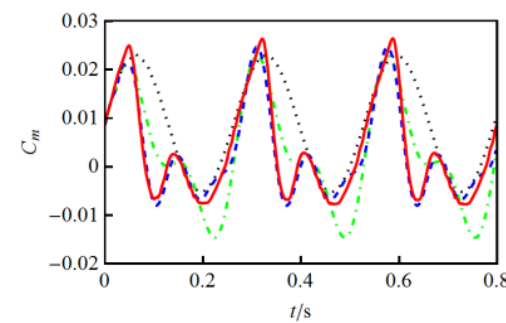

(c) $C_{m}$

Figure 6. Aerodynamics in plunge motion when angle of attack $\alpha_{0}=1^{\circ}$ and reduced frequency $k=0.05$ 


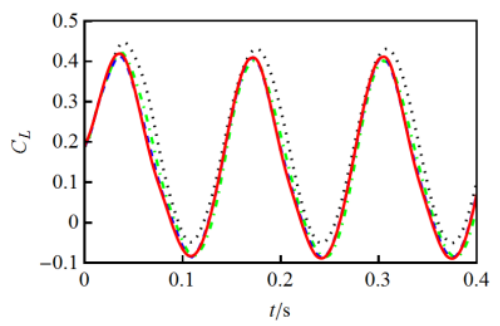

(a) $C_{L}$

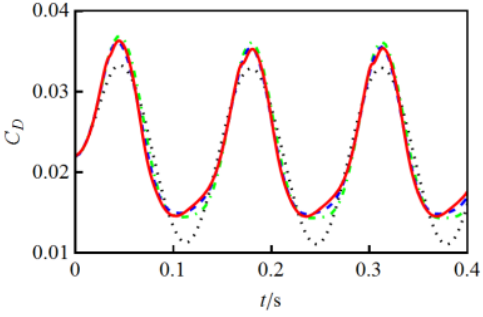

(b) $C_{D}$

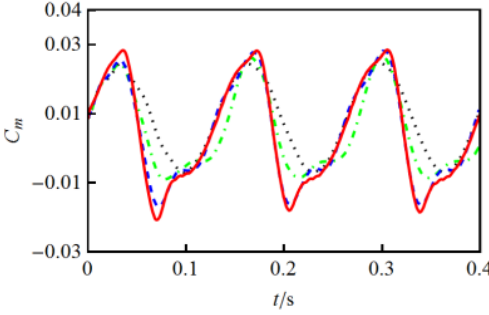

(c) $C_{m}$

$$
\cdots \cdots \cdot h_{1}=-=-h_{1}+h_{2} \cdots \cdots-h_{1}+h_{2}+h_{3} \longrightarrow \text { CFD }
$$

Figure 7. Aerodynamics in plunge motion when angle of attack $\alpha_{0}=1^{\circ}$ and reduced frequency $k=0.1$

\section{Conclusion}

1) In this study, the Volterra series is developed for nonlinear unsteady aerodynamics modeling. The first-order Volterra kernel can only describe the linear characteristics of the system. It is critical to incorporate the influence of higher-order kernels to accurately predict nonlinear aerodynamics.

2) Regarding the difficulties in Volterra kernel identification, the Volterra kernels are expanded with the BSWI as the basis function. The problem is transformed into the solution of low-dimensional equations, and a stable solution is obtained.

3) A nonlinear unsteady aerodynamics model is built by identifying the first three order kernels of the lift, drag, and pitching moment coefficients of the NACA0012 airfoil in plunging motion at transonic speed. The aerodynamics at different reduced frequencies computed by the Volterra series and CFD verify the ability of the Volterra series to predict nonlinear and unsteady aerodynamics.

The aerodynamics modeling in this study focused on the plunge motion. At this time, only the angle of attack changes, and this is a single variable situation. Actually, aerodynamics have multiple influencing factors such as pitch rate. The next step is to investigate the application of multivariate Volterra series methods in unsteady aerodynamics modeling.

\section{References}

1. M. Ghoreyshi, K. J. Badcock, A. D. Ronch, et al., J Aircr, Framework for establishing limits of tabular aerodynamic models for flight dynamics analysis $\mathbf{4 8 ,}$ 42-55 (2011)

2. M. Ghoreyshi, A. Jirasek, R. M. Cummings, AIAA J, Computational investigation into the use of response functions for aerodynamic-load modeling $\mathbf{5 0}$, 1314-1327 (2012)

3. A. J. Mccracken, D. J. Kennett, K. J. Badcock, et al., AIAA Paper 2013-4978, Assessment of tabular models using CFD (2013)

4. M. Ghoreyshi, A. Jirasek, R. M. Cummings, Prog Aerosp Sci, Reduced order unsteady aerodynamic modeling for stability and control analysis using computational fluid dynamics 71, 167-217 (2014)

5. A. D. Ronch, K. J. Badcock, A. Khrabrov, et al.,
AIAA Paper 2011-6524, Modeling of unsteady aerodynamic loads (2011)

6. D. J. Lucia, P. S. Beran, W. A. Silva, Prog Aerosp Sci, Reduced-order modeling: New approaches for computational physics 40, 51-117 (2004)

7. S. Boyd, L. O. Chua, C. A. Desoer, Ima Journal of Mathematical Control and Information, Analytical foundations of Volterra series 1, 243-282 (1984)

8. T. Skujins, C. Cesnik, AIAA Paper 2010-8127, Reduced-order modeling of hypersonic vehicle unsteady aerodynamics (2010)

9. W. A. Silva, AIAA Paper 1999-1262, Reduced-order models based on linear and nonlinear aerodynamic impulse responses (1999)

10. M. Balajewicz, E. Dowell, J Aircr, Reduced-order modeling of flutter and limit-cycle oscillations using the sparse Volterra series 49, 1803-1812 (2012)

11. W. A. Silva, J Aircr, Application of nonlinear systems theory to transonic unsteady aerodynamic responses 30, 660-668 (1993)

12. M. Balajewicz, F. Nitzsche, D. Feszty, AIAA Paper 2009-2319, Reduced order modeling of nonlinear transonic aerodynamics using a pruned Volterra series (2009)

13. C. M. Cheng, Z. K. Peng, W. M. Zhang, et al., Nonlinear Dyn, Wavelet basis expansion-based Volterra kernel function identification through multilevel excitations 76, 985-999 (2014)

14. R. J. Prazenica, A. J. Kurdila, Nonlinear Dyn, Multiwavelet constructions and Volterra kernel identification 43, 277-310 (2006)

15. M. Schetzen, The Volterra and Wiener theories of nonlinear systems (2006)

16. S. Boyd, L. O. Chua, IEEE Trans Circ Syst, Fading memory and the problem of approximating nonlinear operators with Volterra series 32, 1150-1161 (1985)

17. M. Brenner, Y. Jiang, Y. S. Xu, Adv Comput Math, Multiparameter regularization for Volterra kernel identification via multiscale collocation methods $\mathbf{3 1}$, 421-455 (2009)

18. J. W. Xiang, X. F. Chen, Z. J. He, et al., Comput Mech, A new wavelet-based thin plate element using B-spline wavelet on the interval 41, 243-255 (2008)

19. L. Tronchin, J Audio Eng Soc, The emulation of nonlinear time-invariant audio systems with memory by means of Volterra series 60, 984-996 (2012)

20. E. Morelli, S. Derry, M. Smith, AIAA Paper 2005-5921, Aerodynamic parameter estimation for the X-43a (Hyper-X) from flight data (2005) 